\title{
Localization of Dirac-like excitations in graphene in the presence of smooth inhomogeneous magnetic fields
}

\author{
Pratim Roy, Tarun Kanti Ghosh, Kaushik Bhattacharya \\ Department of Physics, Indian Institute of Technology-Kanpur, Kanpur-208 016, India
}

(Dated: October 22, 2018)

\begin{abstract}
The present article discusses magnetic confinement of the Dirac excitations in graphene in presence of inhomogeneous magnetic fields. In the first case a magnetic field directed along the $z$ axis whose magnitude is proportional to $1 / r$ is chosen. In the next case we choose a more realistic magnetic field which does not blow up at the origin and gradually fades away from the origin. The magnetic fields chosen do not have any finite/infinite discontinuity for finite values of the radial coordinate. The novelty of the two magnetic fields is related to the equations which are used to find the excited spectra of the excitations. It turns out that the bound state solutions of the two-dimensional hydrogen atom problem are related to the spectra of graphene excitations in presence of the $1 / r$ (inverse-radial) magnetic field. For the other magnetic field profile one can use the knowledge of the bound state spectrum of a two-dimensional cut-off Coulomb potential to dictate the excitation spectra of graphene. The spectrum of the graphene excitations in presence of the inverse-radial magnetic field can be exactly solved while the other case cannot be. In the later case we give the localized solutions of the zero-energy states in graphene.
\end{abstract}

PACS numbers: 81.05.ue, 03.65.Pm, 73.20.-r, 71.70.Di

\section{INTRODUCTION}

In recent years graphene, which is a single layer of carbon atoms on a honeycomb lattice, has generated a lot of interest in the research community [1 $\left[\begin{array}{l}3 \\ ]\end{array}\right]$. This is essentially because of the experimental realization of graphene sheets in various laboratories and its possible applications in nanoscale devices. An electron in a graphene layer is described by a massless two-dimensional (2D) Dirac-like equation which produces a gapless linear energy spectrum close to the Dirac points $K$ and $K^{\prime}$. The Dirac electron imitates various relativistic phenomena like Klein tunneling [4], unconventional Hall effect [1, 2, 5] and Zitterbewegung [6]. To make electronic devices, the first thing is to confine the electrons. This can be attempted by either using an electric field or a magnetic field. Although zero-energy bound states or quasi-bound states may be created using electrostatic barriers or external potentials, the Dirac electron can not be always confined in this way due to its massless nature [7]. Particularly, the electrostatic barrier becomes completely transparent for normal incidence of the electrons.

Some schemes have been proposed to tackle the problem of confining the electrons. For example, A. de Martino et al., has given an interesting proposal to confine the electron based on an inhomogeneous magnetic field [8]. Later on various inhomogeneous magnetic field configurations have been used in an attempt to confine the electrons or create bound states [9]. Among the various field configurations, exponentially decaying magnetic fields [10], Gaussian magnetic field [11], square well magnetic barrier [8], non-zero magnetic fields in a circular dot [12], magnetic fields corresponding to various solvable potentials [13] etc., have been considered. In all these studies, discontinuous and/or inhomogeneous (in some specific direction) magnetic field profiles were con- sidered.

Recently, Masir et al. 7] has considered a finite-size magnetic dot with a constant magnetic field $\left(B_{z}\right)$ within a circular region with radius $R$, otherwise zero i. e. $\mathbf{B}=B_{z} \Theta(\mathbf{R}-\mathbf{r}) \hat{z}$. It is found that bound state does not exist in this configuration. In the present article our objective is to use different types of axial magnetic fields, which are continuous for any finite radial distance, to examine the possibility of creating bound states in graphene. In the first case we will consider the inverseradial field pointed along the negative $z$-axis and whose magnitude is $\sim 1 / r$. One can exactly solve for the Dirac electron-like excitations in graphene in presence of such a magnetic field. We will focus on the localized excitations in the present case. One of the surprising outcomes of the above analysis is an interesting relation between the bound state spectrum of the 2D Hydrogen atom and the energy spectrum of the magnetically confined graphene excitations. In this article it will be seen that the Schrödinger equation of the 2D Hydrogen atom plays a very important role for determining one of the components of the Dirac-like excitations of graphene in presence of the inverse-radial magnetic field. Due to the complex nature of the Dirac equation it turns out that the relevant Coulomb potential in the 2D Hydrogen atom problem becomes angular momentum dependent. Except this complication of an angular momentum dependent Coulomb potential the 2D Hydrogen atom bound state spectra gives us a clue of the bound state energies of the graphene excitations in an inverse-radial magnetic field.

Although for an inverse-radial magnetic field one can find the spectrum of the localized massless excitations of graphene exactly, still the magnetic field profile is not realistic near the origin. Noting this fact, in the second case, we try to see the properties of the localized excitations in graphene in a more realistic magnetic field by 
introducing a cut-off parameter (i.e, the magnetic field does not blow up at the origin). As a consequence of introducing this cut-off parameter the problem does not remain an exactly solvable one. Nevertheless localized zero-energy states can be exactly determined and it will be seen that in this case also the zero-energy states are infinitely degenerate.

Before we list the materials of the present article in the last paragraph it is useful to give a brief discussion about the magnetic field profiles used in the article. The authors are aware of the fact that the kinds of magnetic fields used for the calculations are not generally used in laboratories. But in the future one may produce a magnetic field which has a high but finite strength in the axial direction and slowly the field strength falls as one moves outwards from the axis. Using various cylindrical dipolar magnets, with the magnetic axis along the cylindrical axis, with varying magnetic strength one can in principle produce a field which can resemble the realistic magnetic field with a cylindrical symmetry as assumed in this article. The central solid cylindrical magnet with the highest magnetic strength must be concentric with many annular cylindrical magnets with lower and lower magnetic strength. The magnetic strengths can be imparted suitably in the laboratories. With two such set ups placed one above the other, such that the opposite poles face each other one can produce magnetic fields of various cylindrical symmetries in the intervening region.

The materials in this article are presented in the following manner. A brief and concise scheme of the mathematical framework which will be utilized throughout the article is presented in the next section. The spectrum of the localized excitations of graphene in the presence of an inverse-radial magnetic field is given in section III. In section [IV we present the exact zero-energy solutions for the graphene excitations in presence of a more realistic magnetic field which does not diverge at the origin. The last section $\mathbf{V}$ concludes the article with a brief discussion and summarizes the findings presented in the article.

\section{THE MODEL}

The quasi-particles in graphene follow a Dirac-like Hamiltonian given by

$$
H=v_{F} \boldsymbol{\sigma} \cdot \mathbf{P},
$$

where $v_{F} \approx 10^{6} \mathrm{~m} / \mathrm{s}$ is the Fermi velocity, $\boldsymbol{\sigma}=\left(\sigma_{x}, \sigma_{y}\right)$ are the Pauli matrices and $\boldsymbol{P}=\boldsymbol{p}+\boldsymbol{A}$ is the canonical momentum with vector potential A. For the sake of simplicity as well as clarity we will use the convention in which $\hbar=e=1$, where $e$ stands for the magnitude of the electronic charge. The eigenvalue equation for the above Hamiltonian is $H \psi(x, y)=E \psi(x, y)$ where $\psi(x, y)$ is a two-component spinor given by

$$
\psi=\left(\begin{array}{l}
\psi_{1}(x, y) \\
\psi_{2}(x, y)
\end{array}\right)
$$

Here the two components $\psi_{1,2}(x, y)$ denote the electron amplitude on two sites in the unit cell of a honeycomb lattice. We now write the eigenvalue equation in the following coupled form as

$$
\Pi_{-} \psi_{2}=\epsilon \psi_{1}, \quad \Pi_{+} \psi_{1}=\epsilon \psi_{2},
$$

where $\epsilon=E / v_{F}$ and $\Pi_{ \pm}=P_{x} \pm i P_{y}$. The general form of the vector potential is of the form

$$
A_{x}=y f(r), \quad A_{y}=-x f(r),
$$

where the function $f(r)$ will be specified later. For this choice of the vector potential the associated magnetic field is perpendicular to the plane and it is given by

$$
B_{z}=-2 f(r)-r f^{\prime}(r) .
$$

Since the magnitude of the magnetic field only depends on $r$ it is convenient to use the plane polar coordinates in the present case. In plane polar coordinates one can write the component spinors as

$$
\psi_{1}=r^{-1 / 2} e^{i m \theta} \chi_{1}(r), \quad \psi_{2}=r^{-1 / 2} e^{i(m+1) \theta} \chi_{2}(r)
$$

where $m=0, \pm 1, \pm 2, \cdots$, stands for the angular momentum quantum number of the pseudo-spinor solution of the Dirac equation. Using the above solutions and Eq. (2) the eigenvalue equation for $\chi_{1}$ can be written as

$$
\left[-\frac{d^{2}}{d r^{2}}+V_{1}^{\mathrm{eff}}(r)\right] \chi_{1}=\epsilon^{2} \chi_{1}
$$

where the effective potential term is

$$
V_{1}^{\mathrm{eff}}(r)=V_{1}(r)+\frac{m^{2}-\frac{1}{4}}{r^{2}},
$$

and

$$
V_{1}(r)=r^{2} f^{2}-2 m f-2 f-r f^{\prime},
$$

where the other term in the potential $V_{1}^{\text {eff }}(r)$ is due to the centrifugal barrier. The factor of $1 / 4 r^{2}$ appears because of the presence of $r^{-1 / 2}$ in the solutions in Eq. (5). Similarly for $\chi_{2}$ we obtain

$$
\left[-\frac{d^{2}}{d r^{2}}+V_{2}^{\mathrm{eff}}(r)\right] \chi_{2}=\epsilon^{2} \chi_{2}
$$

where the effective potential is

$$
V_{2}^{\mathrm{eff}}(r)=V_{2}(r)+\frac{\left(m+\frac{1}{2}\right)\left(m+\frac{3}{2}\right)}{r^{2}},
$$

and

$$
V_{2}(r)=r^{2} f^{2}-2 m f+r f^{\prime} .
$$

It is easy to see that the above equations are standard radial Schrödinger equations (in 2D) with the effective potentials $V_{1,2}^{\text {eff }}(r)$. 


\section{THE INVERSE-RADIAL MAGNETIC FIELD AND THE SPECTRUM OF THE EXCITATIONS}

In this section we will concentrate on a magnetic field profile which is given by

$$
B_{z}=-\frac{\lambda}{r}
$$

where $\lambda>0$ is a constant with the dimension of inverse length. In normal units the magnetic field will be represented as $-\hbar \lambda / e r$. A field of the above type can be obtained by choosing the $f(r)$ as

$$
f(r)=\frac{\lambda}{r}
$$

With the above form of $f(r)$ one can see that

$$
V_{1}(r)=V_{2}(r)=-\frac{\lambda(2 m+1)}{r}+\lambda^{2}
$$

It is interesting to note that with the form of $f(r)$ as

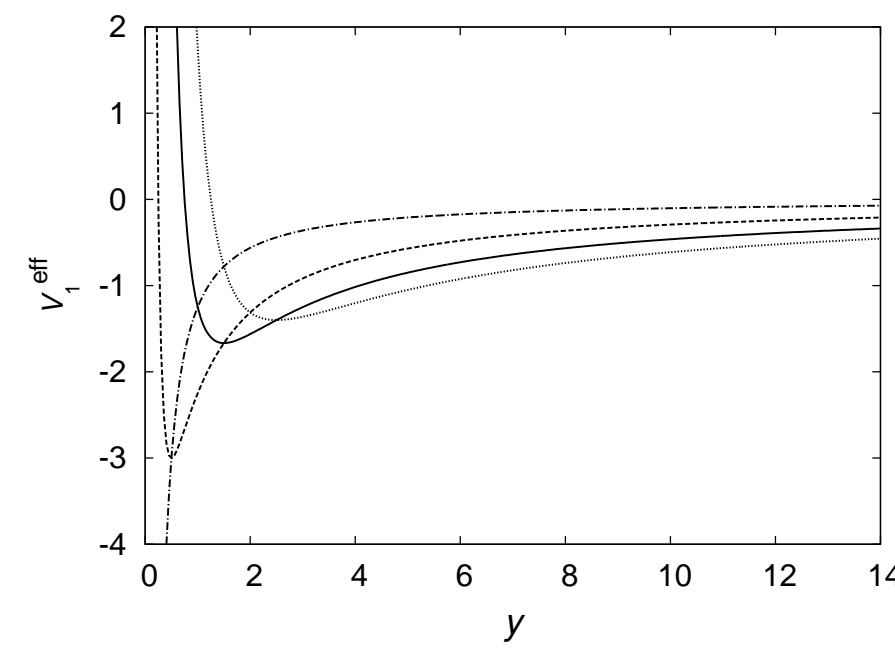

FIG. 1: Plots of the effective potential $V_{1}^{\text {eff }}$ vs $y$ for $m=0$ (dot-dashed), $m=1$ (dashed), $m=2$ (solid) and $m=3$ (short-dashed).

given in Eq. (13), both the potentials $V_{1,2}(r)$ turns out to be of the Coulomb-type for $m \geq 0$. The shape of the effective potentials $V_{1,2}^{\text {eff }}(r)$ for some positive values of $m$ are plotted in Fig. 11 and Fig. 2. The shape of the effective potentials clearly shows that there can be bound states for positive values of the angular momentum quantum numbers. For $m<0$ the potentials $V_{1,2}(r)$ are not attractive and hence do not support any bound state. Consequently, for states with $m \geq 0$ the Dirac electron-like excitations in graphene due to the inverseradial magnetic field can be understood by solving the radial Schrödinger equation, Eq. (6), in presence of a $2 \mathrm{D}$ Coulomb potential. The novelty of the $2 \mathrm{D}$ Coulomb potential, appearing in the Schrödinger equations, in the

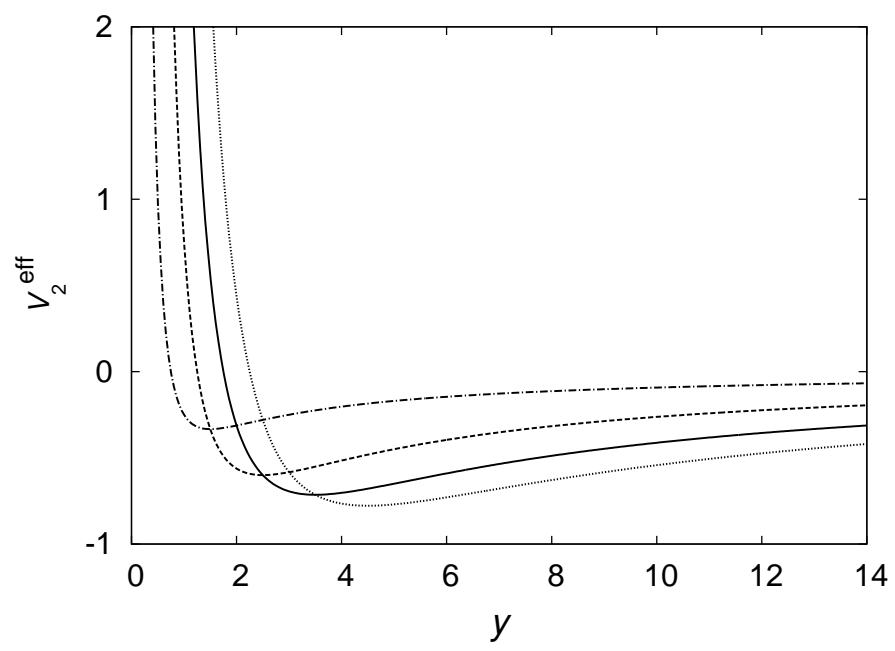

FIG. 2: Plots of the effective potential $V_{2}^{\text {eff }}$ vs $y$ for $m=0$ (dot-dashed), $m=1$ (dashed), $m=2$ (solid) and $m=3$ (short-dashed).

present case is related to the fact that the potentials depends on the angular momentum of the excitations. This observation is pivotal for the analysis which follows.

\section{A. The 2D Hydrogen atom and the upper component of the graphene pseudospinor}

To find the ground state and excited state energies and the corresponding wave functions of the Dirac electronlike excitations in graphene in presence of an inhomogeneous magnetic field, as given in Eq.(12), we require to solve Eq. (6) where $V_{1}(r)$ in the effective potential is given in Eq. (14). One can write Eq. (6) in terms of the function

$$
\phi_{1}(r)=r^{-1 / 2} \chi_{1}(r)
$$

as

$$
-\frac{d^{2} \phi_{1}}{d y^{2}}-\frac{1}{y} \frac{d \phi_{1}}{d y}+\left[-\frac{2 m+1}{y}+\frac{m^{2}}{y^{2}}\right] \phi_{1}=\xi \phi_{1},
$$

where

$$
y \equiv \lambda r,
$$

is a dimensionless parameter and

$$
\lambda^{2} \xi=\epsilon^{2}-\lambda^{2} .
$$

The energy eigenvalues are given by

$$
E= \pm \lambda v_{F} \sqrt{1+\xi} \text {. }
$$

Eq. (16) can be converted to a confluent hypergeometric equation in $x$ where

$$
y=\frac{1}{\beta_{N}} x, \quad \beta_{N}=\frac{2 m+1}{N},
$$


for a real number $N$. Defining a new function $\Phi_{1}(x)$ as

$$
\phi_{1}(x)=x^{m} e^{-x / 2} \Phi_{1}(x),
$$

Eq. (16) transforms into the following confluent hypergeometric differential equation:

$x \frac{d^{2} \Phi_{1}}{d x^{2}}+[(2 m+1)-x] \frac{d \Phi_{1}}{d x}-\left(-N+m+\frac{1}{2}\right) \Phi_{1}=0$.

The most general solution of the above equation is

$$
\begin{aligned}
\Phi_{1}(x) & =A_{1} F_{1}\left(-N+m+\frac{1}{2}, 2 m+1, x\right) \\
& +B U\left(-N+m+\frac{1}{2}, 2 m+1, x\right),
\end{aligned}
$$

where ${ }_{1} F_{1}\left(-N+m+\frac{1}{2}, 2 m+1, x\right)$ and $U(-N+m+$ $\left.\frac{1}{2}, 2 m+1, x\right)$ forms a pair of linearly independent solutions of the confluent hypergeometric equation. $A$ and $B$ are two arbitrary constants.

In our analysis of $\phi_{1}(x)$ the angular quantum number $m$ can be zero or positive integers. The nature of $N$ will dictate the properties of the confluent hypergeometric functions. Out of various possibilities of choosing $N$ one is particularly interesting. If $N$ are half-integers as

$$
N=\frac{1}{2}, \frac{3}{2}, \frac{5}{2}, \cdots,
$$

then $-N+m+\frac{1}{2}$ is a negative integer or zero if we restrict the $m$ values in an appropriate way. Defining

$$
n=N+\frac{1}{2}=1,2,3, \cdots
$$

the possible $m$ values for which $-N+m+\frac{1}{2}$ can be negative or zero turns out to be

$$
m=0,1,2, \cdots, n-1 .
$$

When $-N+m+\frac{1}{2}$ or $-n+m+1$ is a negative integer or zero then ${ }_{1} F_{1}(-n+m+1,2 m+1, x)$ reduces to a finite polynomial in the positive $x$ range. On the other hand if $-n+m+1$ is a negative integer then $U(-n+m+1,2 m+1, x)$ and ${ }_{1} F_{1}(-n+m+1,2 m+1, x)$ are not linearly independent of each other and consequently the pair of functions in Eq. (20) does not give the most general solution of Eq. (19). In this special case one may think of the second solution of Eq. (19) as $e^{x} U(m+n, 2 m+1,-x)$ [17], which is linearly independent of ${ }_{1} F_{1}(-n+m+1,2 m+1, x)$. But, the function $e^{x} U(m+n, 2 m+1,-x)$ diverges at large values of $x$ and consequently this second solution is also unsuitable in the most general solution of Eq. (19).

If the second solution of the confluent hypergeometric equation is omitted then the solution of Eq. (19) coincides with the solution of the $2 \mathrm{D}$ hydrogen atom [18]. In this case

$$
\begin{aligned}
\phi_{1}^{(n, m)}(y) & =\left(\beta_{n} y\right)^{m} e^{-\beta_{n} y / 2} \\
& \times\left[{ }_{1} F_{1}\left(-n+m+1,2 m+1, \beta_{n} y\right)\right]
\end{aligned}
$$

up to a normalization constant and

$$
\beta_{n}=\frac{2(2 m+1)}{2 n-1} .
$$

The eigenvalues in the present case given by $\xi_{n, m}$ will be of the form

$$
\xi_{n, m}=-\frac{(2 m+1)^{2}}{(2 n-1)^{2}}
$$

where $m=0,1,2,3, \cdots$ and $n=1,2,3, \cdots$ the positive integers only. From the expression of the energy eigenvalues as given in Eq. (18) we get

$$
E_{n, m}= \pm \lambda v_{F} \sqrt{1-\frac{(2 m+1)^{2}}{(2 n-1)^{2}}}
$$

The negative energies in the above represent the holes in the valence band. The exact energy eigenvalues of the Dirac electron-like excitations in graphene in presence of an inhomogeneous magnetic field is seen to depend on two quantum numbers. One of them is the radial one, $n$, while the other is related to the angular momentum quantum number $m$.

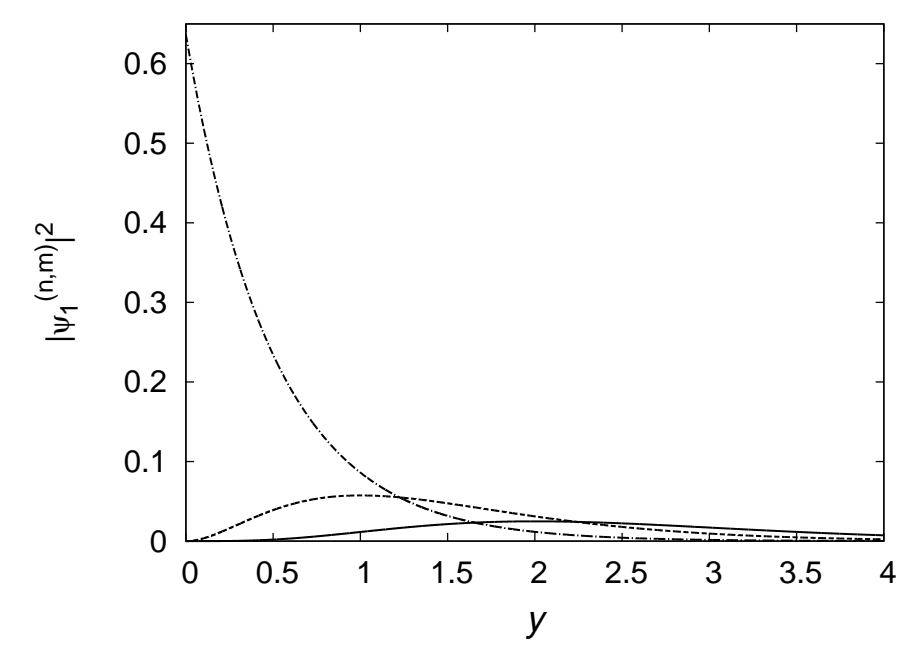

FIG. 3: Plots of the normalized probability density of the zero-energy states vs $y$ for $n=1, m=0$ (dot-dashed), $n=$ $2, m=1$ (dashed), and $n=3, m=2$ (solid).

\section{B. Degeneracies}

If one looks at the energy spectrum of the excitations one will see that there are states which are degenerate. The degeneracies can be understood from the form of the energy eigenvalues as given in Eq. (18). It can be easily observed that

$$
E_{1,0}=E_{2,1}=E_{3,2}=\cdots=E_{n, n-1}=\cdots=0 .
$$


This implies that the ground states of the excitations are infinitely degenerate. The corresponding wave function is given by

$$
\psi^{(m+1, m)}(r, \theta)=N_{m}\left(\begin{array}{c}
y^{m} e^{-y} e^{i m \theta} \\
0
\end{array}\right),
$$

where $m=0,1,2, \cdots$. The normalization constant is

$$
N_{m}=\sqrt{\frac{1}{2 \pi 2^{2(m+1)} \Gamma(2 m+2)}} .
$$

The probability density plots for some of the zero-energy solutions are given in Fig. 3. All of the curves show that the states are localized near the origin and can be treated as proper bound states.

In general, there are many other infinitely degenerate states. For example, the energy levels are infinitely degenerate if $(2 m+1) /(2 n-1)=p / q$, where $p$ and $q$ are odd integers with $p \leq q$. The infinitely degenerate zeroenergy state is obtained for $q=p$.

\section{The lower component of the pseudospinor}

For excited states, $\epsilon \neq 0$, the lower component of the pseudospinor can be obtained by the relation $\psi_{2}=$ $\frac{1}{\epsilon} \Pi_{+} \psi_{1}$. In the present case $\psi_{1}(y)=e^{i m \theta} \phi_{1}(y)$ and consequently one can write

$$
\psi_{2}(y)=-\frac{i \lambda}{\epsilon} e^{i(m+1) \theta}\left[\frac{d}{d y}-\frac{m}{y}+1\right] \phi_{1}(y),
$$

where $\phi_{1}(y)$ is as given in Eq. (24). But the lower component of the zero-energy states cannot be obtained from the formula given above. To obtain the the lower component of the pseudospinor for the zero-energy case one can directly use the first order equations in Eq. (2). Solving the first order equation $\Pi_{-} \psi_{2}=0$ where $\Pi_{-}=$ $e^{-i \theta}[-i \partial / \partial r-(1 / r) \partial / \partial \theta+i \lambda]$ and $\psi_{2}(r)=\phi_{2}(r) e^{i(m+1) \theta}$ one easily obtains

$$
\phi_{2}(y)=\frac{e^{y}}{y^{m+1}}
$$

up to a normalization constant and where $y=\lambda r$. The solution listed above is not square integrable and consequently it cannot be taken as the lower component of the pseudospinors. Consequently the lower component of the zero-energy states are all zero. The modulus squared pseudospinor components, $\psi_{1,2}^{(n, m)}$, for two specific cases as, $m=0, n=2$ and $m=1, n=3$, are plotted in Fig. 4 and Fig. 5, respectively. Here $\psi_{1,2}^{(n, m)}$ stands for either the upper component $\psi_{1}^{(n, m)}$ or the lower component $\psi_{2}^{(n, m)}$ of the pseudospinor for arbitrary values of the quantum numbers $n$ and $m$. Both the curves show that the probability densities of the excitations in both these cases are localized.

\section{Reversing the direction of the magnetic field}

It is interesting to see what happens to the spectrum of the excited states when the magnetic field direction is reversed. From Eq. (13) it can be seen that the magnetic field direction reversal is equivalent to changing the sign of $\lambda$. When the sign of $\lambda$ changes the potentials $V_{1,2}(r)$ do not remain attractive for $m \geq 0$ and so localized states of the Dirac excitations cannot be obtained. On the other hand for negative angular momentum states, $m \leq-1$, Eq. (14) reveals that an attractive potential is possible when $\lambda<0$. Consequently, when the magnetic field direction is reversed one obtains localized states for the Dirac excitations for $m \leq-1$. From the form of the effective potentials in the Schrödinger equations of $\chi_{1}$ and $\chi_{2}$, as given in the initial part of section II it can be easily verified that if one changes the sign of $\lambda$ and replaces $m$ by $-(m+1)$ (for a negative $m$ where $m \leq-1$ ) then $\chi_{1}$ and $\chi_{2}$ just gets interchanged. Consequently we can say that the effect of the magnetic field reversal amounts to interchanging the components of the pseudospinors previously obtained for the magnetic field pointing in the negative $z$-axis with their positive $m$ values replaced by $-(m+1)$ where now $m \leq-1$. It is interesting to point out that the interchange of the components of the pseudospinors can also be accompanied by some some relative phases between them.

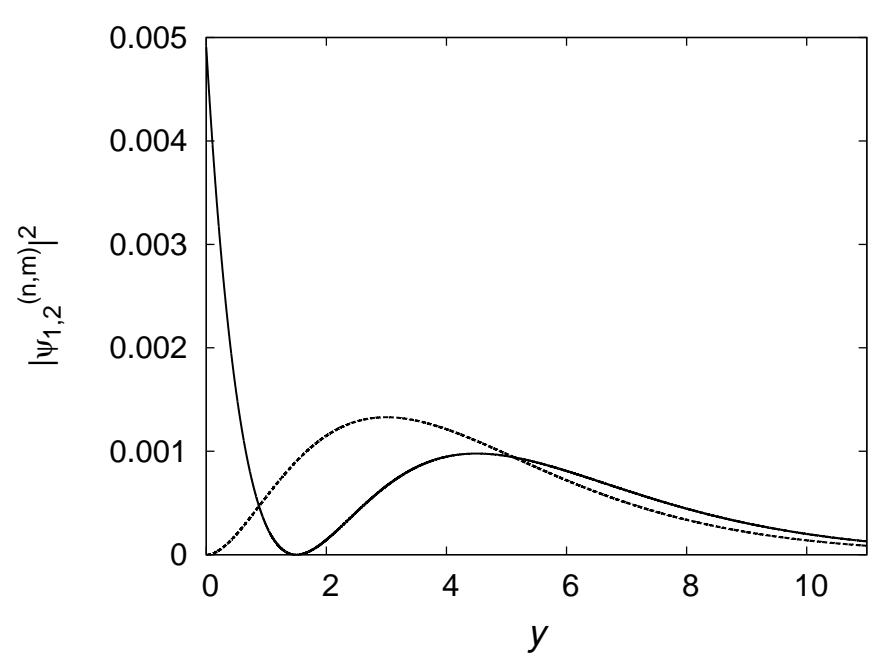

FIG. 4: Plots of the normalized density profile of the upper component (solid) and lower component (dash) for $n=2, m=$ 0 .

\section{THE GROUND STATE SPECTRUM OF A MORE REALISTIC MAGNETIC FIELD}

Till now the present article discussed about a magnetic field profile which becomes infinitely large at around $r=0$. The advantage of choosing such a magnetic field 


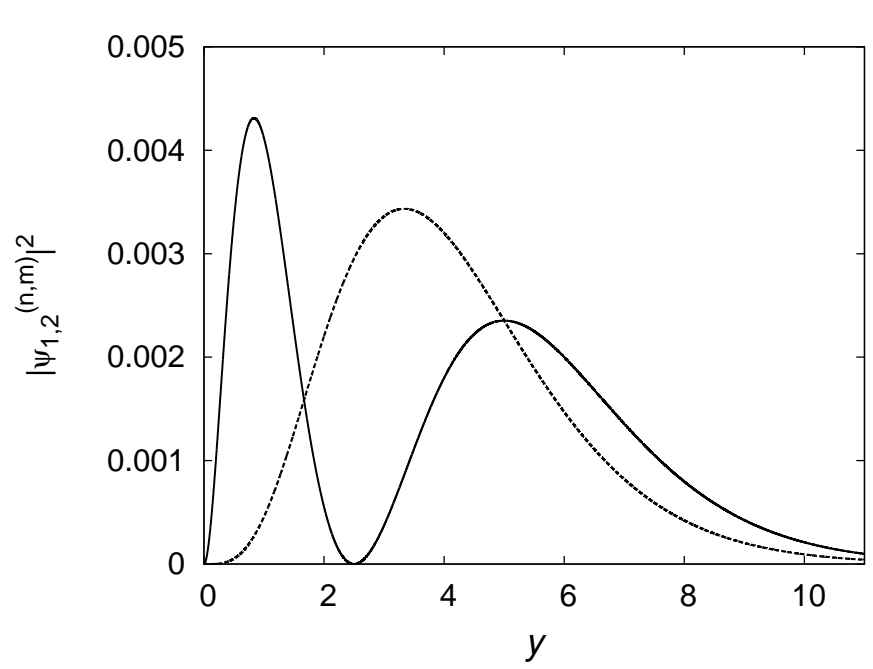

FIG. 5: Plots of the normalized density profile of the upper component (solid) and lower component (dash) for $n=3, m=$ 1.

is related to the fact that the Dirac-like excitations in graphene in the presence of such a field becomes localized and the spectrum of the excitations can be exactly solved. The exact solutions of the previous case could be found because one can match the problem of the graphene excitations in presence of an inhomogeneous magnetic field to the exact solutions of a $2 \mathrm{D}$ hydrogen atom bound state spectrum. In this section we will choose a magnetic field which produces a cut-off Coulomb scalar potential [14, 15] in the effective Schrödinger equation for the upper component $\chi_{1}$. In this section we will mainly focus on the localized ground states of the Dirac excitations which can be exactly found.

If one chooses the function $f(r)$ as

$$
f(r)=\frac{1}{a(r+a)}, \quad a>0,
$$

where $a$ is a positive constant whose actual dimension is that of length then the magnetic field is given by

$$
B_{z}(r)=-\frac{1}{a(r+a)}-\frac{1}{(r+a)^{2}} .
$$

In normal units the magnetic field will be obtained by multiplying the above expression by $\hbar / e$. Clearly the magnetic field is a variable one and finite everywhere. In fact, it lies in the interval $-2 / a^{2} \leq B_{z}(r) \leq 0$. Let us now consider the sector $m \geq 0$. For the above choice of $f(r)$ the scalar potentials $V_{1,2}(r)$ are given by

$$
\begin{aligned}
& V_{1}(r)=-\frac{2 m+3}{a(r+a)}+\frac{1}{a^{2}}, \\
& V_{2}(r)=-\frac{2 m+3}{a(r+a)}+\frac{2}{(r+a)^{2}}+\frac{1}{a^{2}} .
\end{aligned}
$$

In the present case the two scalar potentials are different. It may be noted that apart from the constant $1 / a^{2}$ which may be absorbed in the energy, $V_{1}(r)$ is the cutoff Coulomb potential albeit, in two dimensions. The other potential, $V_{2}(r)$, is not a cut-off Coulomb potential since it contains an additional term. We would like to point out that the cut-off Coulomb potential of the form $V(r)=-\lambda /(r+a)$, where $\lambda>0$, has been extensively studied. The approximate solutions [14] as well as numerical solutions [15] of the Schrödinger equation in an cut-off Coulomb potential have been found using various methods. However in most of these studies the potential is treated in three dimensions where the angular momentum takes positive integer values starting from zero. On the other hand in the present case we are working in two dimensions and the coupling depends on $m$. Thus for $m \geq-1$ the coupling is positive and the potential is an attractive one. On the other hand for $m<-1$ the potential becomes repulsive.

We note that Eq.(6) admits a $\epsilon=0$ solution and then it is given by

$$
\chi_{1}=r^{m+1 / 2}(r+a) e^{-r / a},
$$

up to a normalization constant. Clearly $\chi_{1}(r) \rightarrow 0$ as $r \rightarrow \infty$, consequently $\chi_{1}(r)$ is normalizable.

The lower component of the pseudospinor solution of the Dirac equation can be written down once Eq.(9) is solved. The $\epsilon=0$ solution of Eq.(9) is given by

$$
\chi_{2}(r)=r^{-m-1 / 2} \frac{e^{r / a}}{r+a},
$$

which is clearly non-normalizable for $m \geq 0$. Therefore the $E=0$ solution of the original problem is given by

$$
\psi_{-}^{(m)}(r, \theta)=N_{-}\left(\begin{array}{c}
r^{m} e^{i m \theta}(r+a) e^{-r / a} \\
0
\end{array}\right),
$$

where $m=0,1,2, \cdots$. The normalization constant is

$$
N_{-}=\sqrt{\frac{2^{2 m+3}}{2 \pi a^{2 m+4}(m+3)(2 m+3) \Gamma(2 m+2)}} .
$$

It may be noted that $\psi_{-}^{(m)}(r, \theta)$ is normalizable for all values of the quantum number $m \geq 0$. This means that the $\epsilon=0$ state i.e, $E=0$ state is infinitely degenerate. Furthermore, there is no pseudospin down state corresponding to this level. For the case where $m<0$, neither the solutions in Eq. (37) nor Eq. (38) are normalizable. Consequently for $m<0$ all the states have non-zero energy. The probability density of the zero-energy excitations for various angular momentum values are plotted in Fig. 6. The curves show the localized nature of the states.

\section{A. Reversing the field direction}

For the sake of completeness one can also analyze the spectrum of the Dirac electron-like excitations of 
graphene in the specific magnetic field as given in Eq. (34) with its sign reversed. In this case the vector potentials and the magnetic field are given by

$$
A_{x}=-y f(r), \quad A_{y}=x f(r),
$$

where $f(r)$ is as given in Eq. (33). The zero-energy solutions of Eq. (6) and Eq. (9) with the appropriate gauge potentials as given in Eq. (41) are given as

$$
\chi_{1}=\frac{r^{m+1 / 2}}{r+a} e^{r / a}, \quad \chi_{2}=r^{-(m+1 / 2)}(r+a) e^{-r / a},
$$

up to a normalization constant. From the above expression it is seen that $\chi_{1}$ is not normalizable for any value of $m$. On the other hand, $\chi_{2}$ is also not normalizable for $m \geq 0$. However, for $m<0$ the term $-\left(m+\frac{1}{2}\right)$ becomes positive and consequently $\chi_{2}$ becomes normalizable for $m=-1,-2, \ldots$. In other words when the direction of the magnetic field is reversed, the $E=0$ pseudospin down states are infinitely degenerate and there are no $E=0$ pseudospin up states. Explicitly the $E=0$ solutions are given by

$$
\psi_{+}^{(m)}(r, \theta)=N_{+}\left(\begin{array}{c}
0 \\
r^{-(m+1)} e^{i(m+1) \theta}(r+a) e^{-r / a}
\end{array}\right),
$$

where $m=-1,-2,-3 \cdots$ and $N_{+}$is obtained from $N_{-}$if one replaces the $m$ by $-(m+1)$. Except these replacement in $N_{-}$to obtain $N_{+}$there can be a possible phase factor arising out of the magnetic field reversal about which we will discuss later. The important thing to note

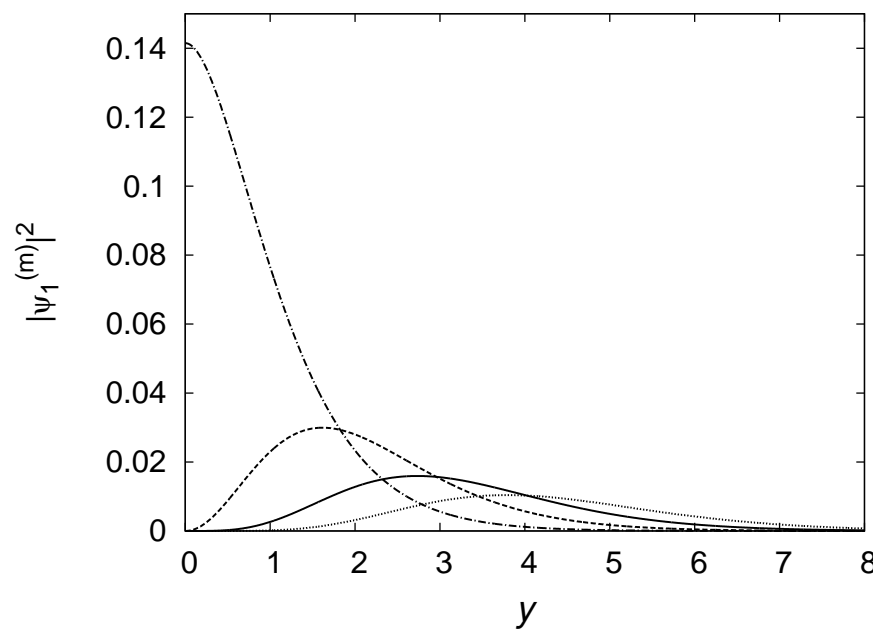

FIG. 6: Plots of the normalized probability density of the zero-energy states vs $y$ for different values of $m=0$ (long dot-dashed), $m=1$ (dashed), $m=2$ (solid) and $m=3$ (short dot-dashed). Here $\psi_{1}^{(m)}$ is the upper component of the pseudospinor for arbitrary values of $m$.

is that when the magnetic field is reversed instead of the pseudospin up state we only have the pseudospin down state in the zero-energy solution.
In this case also it can be noted that if one reverses the magnetic field and replaces $m$ by $-(m+1)$ (for a negative $m$ where $m \leq-1)$ then $\chi_{1}$ and $\chi_{2}$ just gets interchanged. The result of the field reversal essentially remains the same as discussed in the previous section. Magnetic field reversal amounts to interchanging the components of the pseudospinors previously obtained for the magnetic field pointing in the negative $z$-axis with their positive $m$ values replaced by $-(m+1)$. Presently $m \leq-1$ and the interchange of the pseudospinor components can be accompanied by a possible relative phase factor multiplying any of the components.

We would like to note that many of these unusual spectral properties for 2D inverse-radial magnetic fields was also observed in the case of planar Schrödinger equation in the presence of a non-constant magnetic field [16]. In the present case the presence of pseudospin adds additional terms to the effective potential and makes the situation more interesting.

\section{DISCUSSION AND CONCLUSION}

The present article discusses about the possibility of localizing the massless Dirac excitations in graphene in the presence of some specific magnetic field profiles. The unique structure of the minimally coupled Dirac equation for a massless particle in two dimensions gives an interesting physical and mathematical arena to look out for localized excitations. Localization of the excitations in graphene is an important topic as conventional electrostatic methods to localize excited modes fail. The inverse-radial magnetic field, which is directed along the $z$ direction, has the potential to localize the graphene excitations near the origin. While there were many previous works dealing with the topic of magnetic confinement of the excitations in graphene, most of those works used magnetic fields which vanished sharply at some radial distance from the origin. The inverse-radial magnetic field defies this trend of choosing magnetic field profiles which can produce magnetic confinement of the excitations in the sense that the inverse-radial magnetic field does not sharply fall or rise at any distance from the origin. Although the magnetic field gradually falls away from the origin most of the excitations are localized near the origin.

It was pointed out in the article that in presence of the inverse-radial magnetic field the upper component of the pseudospinor of the graphene excitations follow an effective Schrödinger equation. An interesting observation related to the effective Schrödinger equation of the upper component is related to the fact that this equation turns out to be the Schrödinger equation of the $2 \mathrm{D}$ hydrogen atom. Consequently if one knows the bound state spectrum of the 2D Hydrogen atom one can also solve for the upper component of the graphene excitations in presence of an inverse-radial magnetic field. The lower component of the spinor can easily be found by the 
application of the Dirac equation. Our work gives a natural and physical application to the 2D hydrogen atom problem which by itself remains an esoteric and formal problem to deal with.

In the case of the finite magnetic field the relevant Dirac equation for the excitations had a close connection with the 2D cut-off Coulomb potential. The Schrödinger equation for a cut-off Coulomb potential still remains to be analytically solved. Consequently for the realistic magnetic field the full excitation spectrum could not be presented because to present the full spectrum one has to solve an effective Schrödinger equation in presence of the 2D cut-off Coulomb potential.

One of the interesting features of the spectra of the graphene excitations in presence of the inhomogeneous magnetic fields is related to the degeneracy pattern of the solutions. We have tried to point out some of the relevant degeneracy patterns and a general way how these degeneracies arise in the case of the inverse-radial magnetic field. The ground state of the magnetically localized ground state turns out to be infinitely degenerate in both the cases. This is valid when the system size is infinite and the wave function vanishes at infinity. In principle, infinite number of electrons can be accommodated in the zero-energy states. For a realistic situation the degeneracy in the energy levels can not be infinity because of the finite size of the system. Fig. 3 shows that the position of the peak of the densities for various cases is shifting away from the origin as we increase the quantum numbers $(n, m)$. The distance of the point where the density maximizes can not be greater than the radius of the system. This implies that there is an upper cut-off in the $(n, m)$ values. Therefore, the degeneracy is finite in a realistic situation.

Throughout our analysis we have tried to see the effects of a magnetic field direction reversal on the excitation spectrum. The result obtained shows up most prominently in the zero-energy states. When the magnetic field is pointed in the negative $z$-direction only the upper component of the pseudospinor survives where as reversing the field direction kills the upper component and only the lower component of the pseudospinor exists. In the most general case it was pointed out that a magnetic field reversal interchanges the pseudospinor components accompanied by a change of the $m$ quantum numbers to $-(m+1)$ where now $m \leq-1$. It was also pointed out for both the cases, discussed in this article, such an interchange of the pseudospinor components could be accompanied by a relative phase arising out of the field reversal. In this section we can shed some light on the relative phase accompanying such a magnetic field reversal.

It is interesting to connect these phenomenon related to the magnetic field reversal with the time-reversal like operation $\mathcal{S}$ as discussed in the review by Beenakker [20]. Under the action of $\mathcal{S}$ the Hamiltonian of the excitation in Eq. (11) changes as

$$
\mathcal{S} H(\mathbf{A}) \mathcal{S}^{-1}=H(-\mathbf{A}),
$$

where $\mathbf{A}$ is the gauge potential giving rise to the magnetic field. Although $\mathcal{S}$ is not the exact time-reversal operator, the exact time reversal operator changes the valleys, it acts like the time-reversal operator in a single valley. The action of $\mathcal{S}$ is like reversing the magnetic field direction. The operator $\mathcal{S}=i \sigma_{2} C$ where $C$ is the complex conjugation operation. It can easily be verified that if one apply this operator on the pseudospinors one gets the pseudospinors of the reversed field case, as discussed in this article. As a result of the $\mathcal{S}$ operation on the pseudospinors the component of the previous pseudospinor components gets interchanged with a relative sign change. This appearance of a sign can be easily accommodated in the scheme discussed previously where it was pointed out that the interchange of the pseudospinor components can be accompanied by a relative phase.

To conclude this article it can be stated that the present article tries to analyze whether one can have magnetically confined excitations in graphene with inhomogeneous magnetic fields which do not have any finite/infinite discontinuities in the non-zero finite range of the radial coordinate. The magnetic field profiles chosen turns out to be interesting because their specific functional forms allows one to use the results obtained for the bound states of 2D Schrödinger equations in the presence of a Coulomb or a cut-off Coulomb potential. It is observed that there exists an infinite number of localized excitations which are magnetically confined. In the future if one can produce a inverse-radial magnetic field then these exotic localized excitations can be observed experimentally.

\section{Acknowledgments}

We would like to thank Sudeep Bhattacharjee for a discussion on realization of the inverse-radial magnetic field.
[1] K. S. Novoselov, A. K. Geim, S. V. Morozov, D. Jiang, M. I. Katsnelson, I. V. Grigorieva, S. V. Dubonos, and A. A. Firsov, Nature (London) 438, 197 (2005).

[2] Y. Zheng, J. W. Tan, H. L. Stormer, and P. Kim, Nature (London) 438, 201 (2005).

[3] A. H. Castro Neto, F. Guinea, N. M. R. Peres, K. S.
Novoselov, and A. K. Geim, Rev. of Mod. Phys. 81, 109 (2009).

[4] M. I. Katsnelson, K. S. Novoselov, and A. K. Geim, Nature Phys. 2, 620 (2006).

[5] A. M. J. Schakel, Phys. Rev. D 43, 1428 (1991).

[6] M. I. Katsnelson, Euro. Phys. Journal B 51, 157 (2006); 
T. M. Rusin and W. Zawadzki, Phys. Rev. B 76, 195439 (2007); T. M. Rusin and W. Zawadzki, Phys. Rev. B 78, 125419 (2008).

[7] R. R. Hartmann. N. J. Robinson, and M. E. Portnoi, Phys. Rev. B 81, 245431 (2010); C. A. Downing, D. A. Stone, and M. E. Portnoi, preprint arXiv:1105.0891, M. R. Masir, A. Matulis, and F. M. Peeters, Phys. Rev. B 79, 155451 (2009); A. Matulis and F. M. Peeters, Phys. Rev. B 77, 115423 (2008); P. Hewageegna and V. Apalkov, Phys. Rev. B 77, 245426 (2008); ibid B 79, 115418 (2009); P. G. Silvestrov and K. B. Efetov, Phys. Rev. Lett. 98, 016802 (2007).

[8] A. De Martino, L. Dell'Anna, and R. Egger, Phys. Rev. Lett. 98, 066802 (2007); A. De Martino, Solid State Comm. 144, 547 (2007); L. Dell'Anna and A. De Martino, Phys. Rev. B 79, 045420 (2009).

[9] J. M. Pereira, F. M. Peeters, and P. Vasilopolous, Phys. Rev. B 75, 125433 (2007).

[10] T. K. Ghosh, J. Phys: Condens. Matter 21, 045505 (2009).

[11] C. M. Lee, R. C. H. Lee, W. Y. Ruan, and M. Y. Chou, J. Phys: Condens. Matter 22, 355501 (2010).

[12] D. Wang and G. Jin, Phys. Lett. A 373, 4082 (2009).

[13] S. Kuru, J. M. Negro, and L. M. Nieto, J. Phys: Condens. Matter 21, 455305 (2009).
[14] A. Sinha and R. Roychoudhury, J. Phys A 23, 3869 (1990); H. De Mayer, J. Phys. A 23, 1323 (1990); F. M. Fernandez, Phys. Lett. A 160, 511 (1991); R. N. Chaudhury and M. Mondal, Pramana 39, 493 (1992); R. L. Hall, N. Saad, K. D. Sen, and H. Ciftci, Phys. Rev. A 80, 032507 (2009); J. Math. Phys. 51, 022107 (2010); C. W. Clarke, J. Phys. B 30, 2517 (1997).

[15] C. H. Mehta and S. H. Patil, Phys. Rev. A 17, 43 (1978); S. H. Patil, Phys. Rev. 24, 2913 (1981); D. Singh and Y. P. Varshni, Phys. Rev. A 32, 619 (1985); P. P. Ray and K. Mahata, J. Phys. A 22, 3161 (1989); M. Odeh and O. Mustafa, J. Phys. A 33, 7013 (2000).

[16] H. Grosse and J. Stubbe, Lett. Math. Phys. 34, 59 (1995); H. Grosse, A. Martin, and J. Stubbe, Phys. Lett. A 181, 7 (1993).

[17] M. Abramowitz and I. A. Stegun, Handbook of mathematical functions, Dover publication, Inc., New York, (Tenth printing, 1972).

[18] X. L. Yang, S. H. Guo, F. T. Chan, K. W. Wong, and W. Y. Ching, Phys. Rev. A 43, 1186 (1991); M. E. Portnoi, J. Math. Phys. 43, 4681 (2002).

[19] K. S. Gupta and S. Sen, preprint arXiv:0805.3433 3.

[20] C. W. J. Beenakker, Rev. Mod. Phys. 80, 1337 (2008). 